\title{
The complex metabolism of trimethylamine in humans: endogenous and exogenous sources-CORRIGENDUM
}

\author{
JYOTI CHHIBBER GOEL, ANAMIKA GAUR, VARSHA SINGHAL, NEERAJ PARAKH, \\ BALRAM BHARGAVA, AMIT SHARMA
}

https://doi.org/10.1017/erm.2016.6 Published online by Cambridge University Press, 29 April 2016

In the above mentioned article, the authors apologise for the following errors:

On page 2, under the heading 'TMA synthesis and metabolism' the sentence:

Alternatively, TMA can be degraded to dimethylamine (DMA) and formaldehyde by the microbial enzyme trimethylamine dehydrogenase (TMADH) (Refs 37, 38, 39). Similarly, TMAO can be acted upon by TMAO demethylase (TMADM) to form methane and ammonia by some methanogens (Ref. 40) (Fig. 2).

Should read:

Alternatively, TMA can be degraded to methane and ammonia by the microbial enzyme tri-methylamine dehydrogenase (TMADH) (Refs 37, 38, 39). Similarly, TMAO can be acted upon by TMAO demethylase (TDM) to form DMA and formaldehyde by some methanogens (Ref. 40) (Fig. 2).

Figure 2 and its caption is incorrect. The figure and caption should read as follows: 


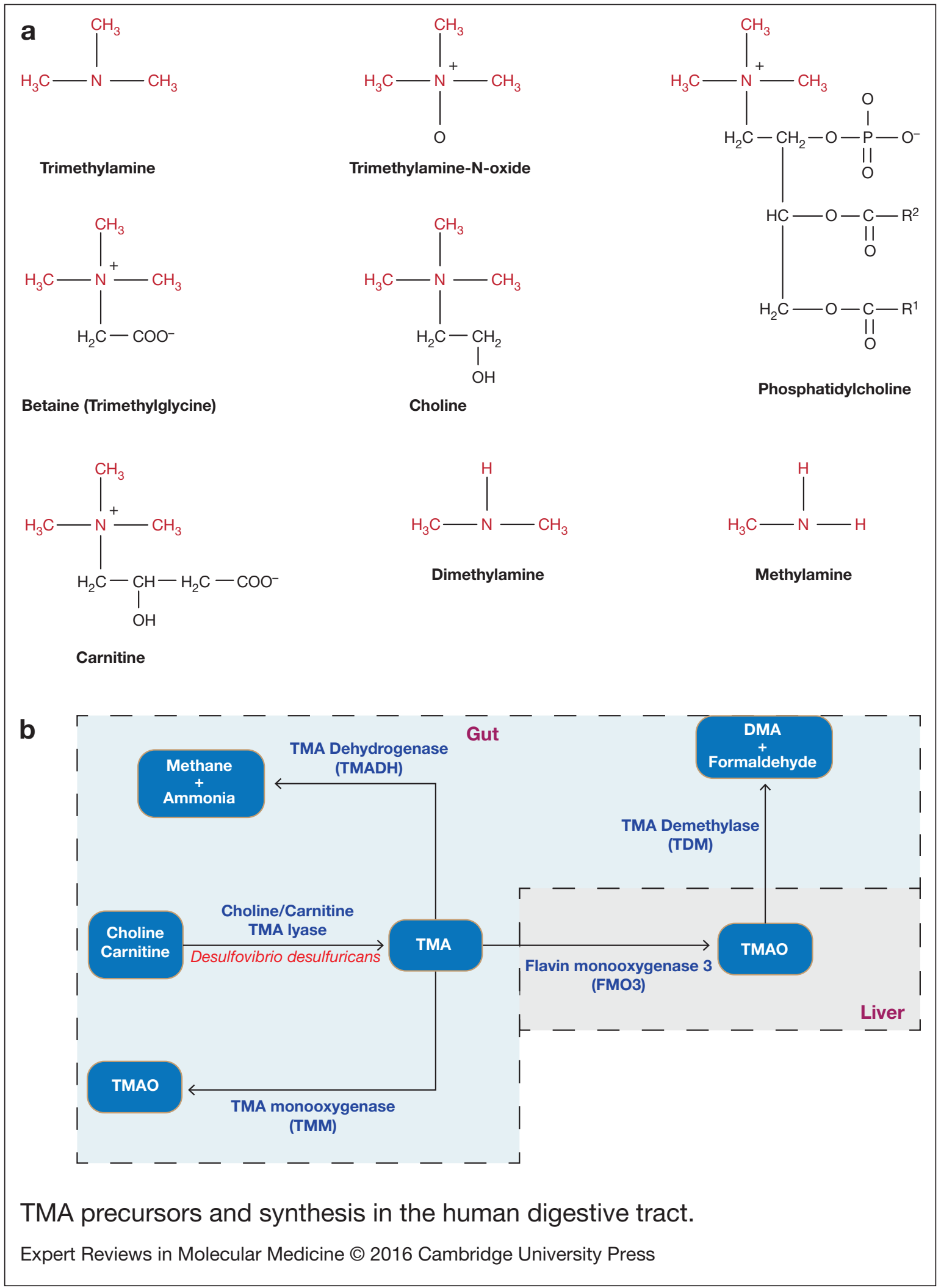

FIGURE 2.

TMA precursors and synthesis in the human digestive tract. (a) Chemical formulae of TMA, its precursors trimethylamine-N-oxide, choline, phosphatidylcholine, carnitine, betaine, and its degradation products DMA and methylamine. (b) Schematic representation of the origin and fate of human gut TMA, which is synthesised using dietary precursors such as choline, carnitine by gut microbial enzymes. Choline TMA lyase acts upon choline leading to the synthesis of TMA (Ref. 116). TMA is then oxidised to TMAO by either mammalian hepatic FMO3 or by the action of microbial TMM (Ref. 33). TMAO can further be degraded to DMA and formaldehyde by the action of TDM (Ref. 40), whereas TMA is metabolised to methane and ammonia by the action of TMADH (Ref. 39). 
Also, figure 5 is incorrect and should read:

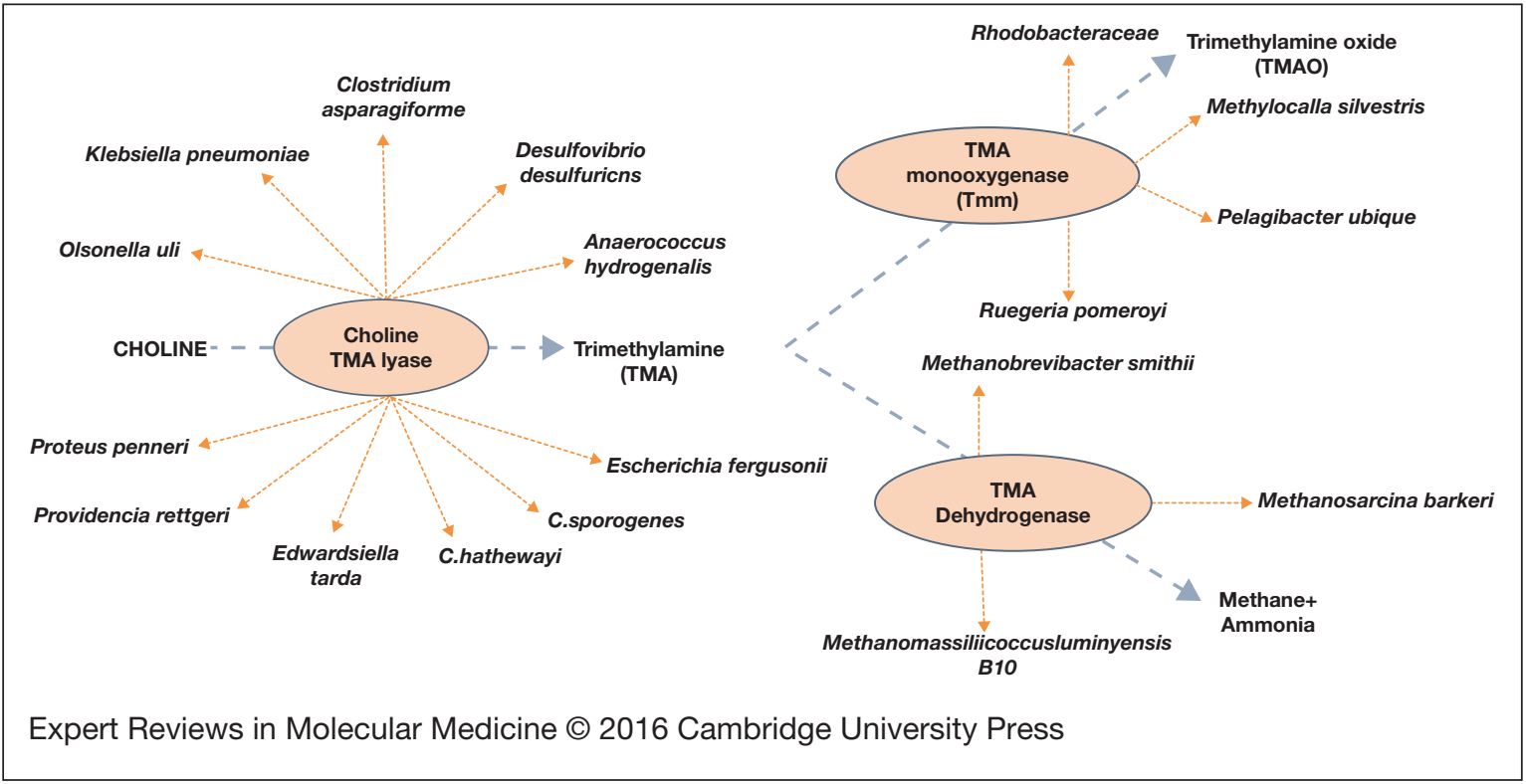

FIGURE 5

Reference

Chhibber-Goel J. et al. (2016) The complex metabolism of trimethylamine in humans: endogenous and exogenous sources. Expert Reviews in Molecular Medicine 18. doi:10.1017/erm.2016.6. 